\title{
Ictal and peri-ictal psychopathology
}

\author{
Marco Mula* and Francesco Monaco \\ Department of Clinical and Experimental Medicine, Amedeo Avogadro University, Division of Neurology, \\ University Hospital Maggiore della Carità, Novara, Italy
}

\begin{abstract}
Patients with epilepsy may experience psychiatric symptoms preceding the seizure (pre-ictal), following the seizure (post-ictal), independently of seizure occurrence (interictal), or as an expression of the seizure (ictal). Compared to interictal, peri-ictal psychiatric symptoms are less investigated and recognized. However, they contribute substantially to disability and distress among people with epilepsy.

The relationship between interictal and peri-ictal symptoms is still largely unknown but it seems that they are intimately related in epilepsy. Greater appreciation and understanding of the peri-ictal period is clinically important, providing a model for understanding basic mechanisms underlying mood and thought disorders and the substrates of cognition, volition, emotion, and consciousness.

The present paper is aimed at reviewing major psychiatric symptoms that may occur around the ictus with special attention to clinical descriptions and relationships with interictal psychopathology.
\end{abstract}

Keywords: Epilepsy, post-ictal psychosis, post-ictal depression, per-ictal dysphoria

\section{Introduction}

Patients with epilepsy may experience a number of psychiatric and cognitive symptoms or behavioral manifestations during the period around the ictus. They are obviously unique to patients with epilepsy and are referred to as peri-ictal symptoms $[11,24]$. These include symptoms preceding (pre-ictal) or following (post-ictal) the seizure or occurring as an expression of the seizure activity (ictal).

The practicality of classifying behavioral symptoms according to their temporal relation to seizure occurrence has been recognized for a long time (Fig. 1). These phenomena have been well described by Gowers [15] and Jackson [17] but also Kraepelin [26] and Bleuler [5] recognized their occurrence in epilepsy. However, among all behavioral manifestations, periictal symptoms still remain the least recognized by clinicians and the least investigated in systematic research

\footnotetext{
* Corresponding author: Dr. Marco Mula MD, PhD, Department of Clinical and Experimental Medicine, Amedeo Avogadro University, Division of Neurology, University Hospital Maggiore della Carità, C.so Mazzini 18, 28100 Novara, Italy. Tel.: +39 03213733371; Fax: +39 03213733298; E-mail: marco.mula@med.unipmn.it.
}

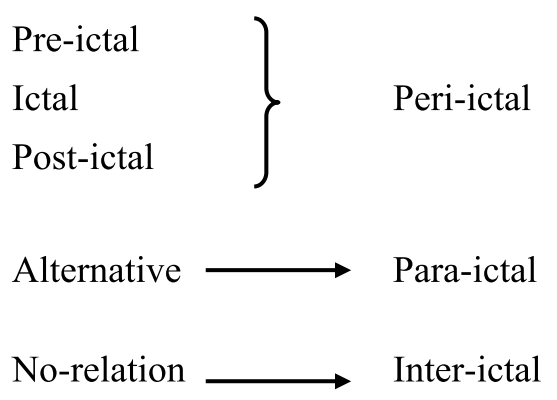

Fig. 1. Classification of psychiatric symptoms according to their temporal relation with seizures.

studies. Such paucity of data probably reflects the attention of clinicians to manifestations strictly connected to cortical EEG epileptic abnormalities, considering all manifestations occurring during an apparently "normal" or aspecific EEG as meaningless. In fact, ictal psychopathology has been better defined and described than peri-ictal phenomena. Nonetheless, peri-ictal psychiatric symptoms contribute substantially to disability and distress among people with epilepsy [6].

This paper is aimed at reviewing and discussing current knowledge about peri-ictal psychiatric symptoms in patients with epilepsy, with special attention to clin- 
ical features and differential diagnosis with interictal psychopathology.

\section{Pre-ictal symptoms}

Pre-ictal psychiatric symptoms usually consist of cluster of symptoms preceding seizures of variable duration, ranging from a few minutes up to three days. Such symptoms, although not characterized by any detectable surface EEG change, probably represent the expression of underlying epileptic activity.

Around one-third of patients with partial seizures report premonitory symptoms, usually before secondary generalized tonic clonic seizures [13]. Prodromal moods of depression or irritability may occur hours to days before a seizure and are often relieved by the convulsion. Blanchet and Frommer [4] investigated the presence of pre-ictal psychiatric symptoms in 27 consecutive patients who were asked to rate their mood on a daily basis for a period of 1 month. Rating scales identified the presence of dysphoric symptoms, consisting of irritability and mood changes, approximately 3 days before the seizures. These symptoms worsened in severity closer to the time of the seizure and remitted approximately 1 day after the seizure, although in some cases symptoms persisted for up to 3 days after the seizure. A recently published study pointed out that behavioral changes are the most frequently reported pre-ictal symptoms, being characterized by irritability or decreased tolerance and lasting several hours [35].

Recently, a prevalence of pre-ictal dysphoric symptoms in patients with epilepsy has been reported. Around $13 \%$ of patients experienced irritability, dysphoria or depressed mood preceding seizures [29]. Such feelings are almost indistinguishable from interictal ones, apart from duration and close relation with seizure occurrence. It seems, therefore, important for clinicians to enquire about these phenomena, because they cannot be detected by rating scales or questionnaires [30].

\section{Ictal symptoms}

In the majority of cases, ictal psychiatric symptoms are very brief (they last less than 30 seconds), stereotyped, occur out of the context and are associated with other seizure-related phenomena such as subtle or overt automatism or post-ictal confusion of variable duration. These symptoms represent simple partial seizures which may be followed by alteration of consciousness as the ictus evolves to a complex partial seizure.

Ictal fear or ictal panic is the most frequently reported ictal psychiatric symptom. It can be a common manifestation of partial seizures originating in right mesial temporal lobe structures [16], representing a simple partial seizure or an aura followed by a complex partial seizure or a secondarily generalized tonic-clonic seizure. Fear is reported as an aura by $10 \%-15 \%$ patients with partial seizures [13]. It seems more common in women than men $[7,38]$ and seems to have a poor prognostic value for surgery [12].

Ictal depression is the second most frequently reported ictal psychiatric symptom. Such mood changes include anhedonia, feeling of guilt and intense suicidal ideation. It occurs as part of an aura in approximately $1 \%$ of patients and more commonly in temporal lobe epilepsy [13].

Ictal psychoses may represent the clinical manifestation of an absence status (generalized non-convulsive status) or mostly a psychomotor status (complex partial status epilepticus) [31]. Patients who experience ictal psychoses may present with either a prolonged twilight or confusional state (continuous form), or a series of recurring seizures with partial responsiveness between seizures (cyclic form) [39]. The duration of symptoms varies from hours to days and EEG shows focal epileptic abnormalities. Still, it is recognized that ictal psychoses of temporal lobe origin are characterized by a more profound impairment of consciousness as compared to extra-temporal ones where speech disturbances and confusion are fluctuating [39].

Ictal hallucinations are usually visual (less frequently auditory). Compared to psychotic hallucinations patients are aware that such symptoms reflect unreal phenomena. It has been stated that ictal hallucinations rising from the insular cortex are associated with a strong autonomic component. Devinsky et al. reported a peculiar type of ictal hallucinations, namely autoscopic or out of the body experiences [8]. The authors pointed out that in the majority of cases they have a localizing value ( $86 \%$ from the temporal lobe) but with no relevance for lateralization.

\section{Post-ictal symptoms}

Post-ictal symptoms may begin either immediately after the seizure (immediate post-ictal period) or more characteristically from 12 to 120 hours after a seizure or a cluster of seizures (also known as lucid interval). 
Post-ictal psychoses are the best characterized phenomena among all peri-ictal manifestations. Historically, it was accepted that patients could develop psychoses in the context of the post-ictal state, the features of which were often manic or hypomanic, although more generally the presentation was one of a mixed mood episode with psychotic features. Original descriptions date back to Esquirol, Jackson and Gowers, however, post-ictal psychoses did not become the focus of attention until much later [28,34]. They represent the most frequent psychiatric manifestation in clinical practice accounting for as much as $25 \%$ of cases of psychosis in one early epilepsy series [9]. Variables linked to post-ictal psychoses in different case series can be summarized as: delay between the onset of psychiatric symptoms and the time of last seizure; relatively short duration of symptoms; strong affective component in the phenomenology; secondary generalized tonic-clonic seizures preceding the onset of postictal psychosis; long duration of the epilepsy; a prompt response to very low dose of antipsychotic drugs and benzodiazepines [10,21].

Oshima et al. [33] pointed out that post-ictal psychoses should be subdivided into two different subtypes: the nuclear type, with an established clinical picture and the presence of the lucid interval, and the atypical peri-ictal type, usually without the lucid interval and with quite a polymorphic clinical presentation. The former probably represents an indirect aftereffect of seizure activity whereas the latter is a direct manifestation of limbic discharge [33]. Kanemoto et al. [18] stressed the typical clinical phenomenology of post-ictal psychosis which seems to be characterized by grandiose delusions as well as religious delusions in the setting of a markedly elevated mood and the feeling of mystic fusion of the body with the universe. In addition, post-ictal psychoses seem to exhibit few schizophreniform psychotic traits such as perceptual delusions or voices commenting, and well-directed violent and self-destructive behavior seem a specific hallmark [20]. Post-ictal psychosis appeared to be a predictor of bilateral ictal foci [22] and consequently a poor surgery outcome [19].

Post-ictal mood changes are less recognized in psychiatric practice as compared to post-ictal psychoses. However, they can be frequently reported by patients and relatives. A case series in a monitoring unit reported $18 \%$ of patients had at least five symptoms of depression lasting more than 24 hours but less than 2 weeks (required by DSM-IV for a major depressive episode) [25]. A comparison of seizure-related vari- ables between these subjects and patients without any post-ictal psychiatric symptom failed to reveal any differences, although patients with post-ictal depression seem to be more likely to have a previous history of psychiatric disorders [24].

Manic/hypomanic symptoms are reported in $22 \%$ of patients, often with associated psychotic phenomenology [25]. It seems that post-ictal mania has a distinct position among mental disorders observed in the postictal period. Such manic episodes last for a longer period and have a higher frequency of recurrence than post-ictal psychoses, being associated with an older age at onset, EEG frontal discharges and dominant hemisphere involvement [32].

Post-ictal anxiety is reported by $45 \%$ patients [25]. The median duration of symptoms ranges from 6 to 24 hours. In one third of cases, post-ictal anxiety may last 24 hours or longer. In about $33 \%$ of cases post-ictal anxiety is reported by patients with a previous history of an anxiety disorder.

\section{Para-ictal symptoms}

Among all psychiatric manifestations that may occur around the ictus those associated with seizure control most need to be taken into account. Landolt reported EEG investigations of patients with epilepsy and paroxysmal psychiatric disorders, describing a group of patients who had productive psychotic episodes with "forced normalization" of the EEG. In other words, abnormal EEGs of these patients improved or normalized during the time that they were psychotic [27]. Subsequently, Tellenbach [37] introduced the term "alternative psychosis" for the clinical phenomenon of the reciprocal relationship between abnormal mental states and seizures, which did not, as Landolt's term did, rely on EEG findings. In any case, since the early observations of Landolt, a number of patients with alternating psychosis have been documented to put their existence beyond doubt and an association with the prescription of antiepileptic drugs was noted [40]. In fact, in many of the series described, the precipitation of the abnormal behavioral state or the psychosis has been linked with the prescription of anticonvulsants but it is important to note that this phenomenon should not be restricted to drug-induced seizure control. In fact, it is likely that in patients who develop de novo psychosis following epilepsy surgery, forced normalization may play such a role, and a case of an alternative psychosis secondary to vagus nerve stimulation has been docu- 
mented [14], suggesting that the mechanisms underlying the control of seizures are strictly interlinked with those of psychosis.

As Landolt himself pointed out, there is no specific clinical presentation of this phenomenon [27]. Of 44 clinical episodes of forced normalization described by Wolf [36], the commonest syndromes were psychosis but in nine cases pre-psychotic dysphoria was described, characterized by insomnia, restlessness, anxiety and social withdrawal. In another two cases there were dysphoric episodes and true manic episodes was also described in two other patients. Wolf made the point that the symptomatology was often determined by personality structure, psychiatric history or familial predisposition.

\section{Relationship between peri-ictal and interictal symptoms}

Relationships between interictal psychopathology and peri-ictal behavioral manifestations are poorly specified. However, emerging evidence clearly suggests that these variables are intimately related, with the majority of supportive data coming from the literature about psychoses in epilepsy.

On one hand, interictal symptoms represent a risk factor for the development of peri-ictal psychiatric symptoms. In fact, Alper et al. [3] showed that a previous history and a family history of any psychiatric disorder may be associated with the development of postictal psychotic episodes. On the other hand, post-ictal psychopathology seems to be connected with the development of a chronic psychiatric disorder. It has been estimated that around $13.9 \%$ of patients with a history of post-ictal psychosis may subsequently develop an interictal psychosis [2,23]. Adachi et al. demonstrated that, in the majority of cases, post-ictal psychotic episodes last less than 1 month but they can be prolonged when individuals have an underlying vulnerability to psychosis [1].

Another interesting issue is that of peri-ictal exacerbation of interictal symptoms. A U.S. study reported exacerbation in the post-ictal period in $94 \%$ of patients and de-novo post-ictal symptoms with different psychopathological features in $83 \%$. Interestingly, the authors pointed out that antidepressant drug treatment did not prevent the development of post-ictal mood symptoms but was shown to be effective for interictal symptoms [11]. Interestingly, it has been suggested that antiepileptic drugs with negative psychotropic properties (e.g. barbiturates and benzodiazepines) may yield a trend toward a greater likelihood of developing post-ictal exacerbation of interictal symptoms [11].

\section{Conclusions}

The presented data illustrate the relatively high prevalence of peri-ictal psychiatric symptoms and their very close relationship with interictal ones. Without doubt, the recognition of these symptoms represents the essence of a thorough appreciation of psychiatric phenomena in epilepsy, including (as it pertains to) the interpretation of its response to treatment. In fact, the failure in identifying such conditions has several practical implications in terms of prognosis and therapeutic strategies.

\section{References}

[1] N. Adachi, M. Ito, K. Kanemoto, N. Akanuma, M. Okazaki, S. Ishida, M. Sekimoto, M. Kato, J. Kawasaki, Y. Tadokoro, T. Oshima and T. Onuma, Duration of postictal psychotic episodes, Epilepsia 48 (2007), 1531-1537.

[2] N. Adachi, M. Kato, M. Sekimoto, I. Ichikawa, N. Akanuma, H. Uesugi, H. Matsuda, S. Ishida and T. Onuma, Recurrent postictal psychosis after remission of interictal psychosis: further evidence of bimodal psychosis, Epilepsia 44 (2003), 1218-1222.

[3] K. Alper, O. Devinsky, L. Westbrook, D. Luciano, S. Pacia, K. Perrine and B. Vazquez, Premorbid psychiatric risk factors for postictal psychosis, J Neuropsychiatry Clin Neurosci 13 (2001), 492-499.

[4] P. Blanchet and G.P. Frommer, Mood change preceding epileptic seizures, J Nerv Ment Dis 174 (1986), 471-476.

[5] E. Bleuler, Lehrbuch der Psychiatrie, pp. viii. 518. Berlin, $1916,8^{\circ}$.

[6] L.S. Boylan, Peri-Ictal Behavioral and Cognitive Changes, Epilepsy Behav 3 (2002), 16-26.

[7] V. Chiesa, E. Gardella, L. Tassi, R. Canger, G. Lo Russo, A. Piazzini, K. Turner and M.P. Canevini, Age-related gender differences in reporting ictal fear: analysis of case histories and review of the literature, Epilepsia 48 (2007), 2361-2364.

[8] O. Devinsky, E. Feldmann, K. Burrowes and E. Bromfield, Autoscopic phenomena with seizures, Arch Neurol 46 (1989), 1080-1088.

[9] S. Dongier, Statistical study of clinical and electroencephalographic manifestations of 536 psychotic episodes occurring in 516 epileptics between clinical seizures, Epilepsia 1 (1959), 117-142.

[10] B. Elliott, E. Joyce and S. Shorvon, Delusions, illusions and hallucinations in epilepsy: 2. Complex phenomena and psychosis, Epilepsy Res 85 (2009), 172-186.

[11] A.B. Ettinger and A.M. Kanner, Psychiatric issues in epilepsy: a practical guide to diagnosis and treatment, Wolters Kluwer Health/ Lippincott Williams and Wilkins, Philadelphia, PA; London, 2007, xxiv, 547. 
[12] M. Feichtinger, E. Pauli, I. Schafer, K.W. Eberhardt, B. Tomandl, J. Huk and H. Stefan, Ictal fear in temporal lobe epilepsy: surgical outcome and focal hippocampal changes revealed by proton magnetic resonance spectroscopy imaging, Arch Neurol 58 (2001), 771-777.

[13] A. Gaitatzis, M.R. Trimble and J.W. Sander, The psychiatric comorbidity of epilepsy, Acta Neurol Scand 110 (2004), 207220.

[14] S.D. Gatzonis, E. Stamboulis, Siafakas, E. Angelopoulos, N. Georgaculias, E. Sigounas and A. Jekins, Acute psychosis and EEG normalisation after vagus nerve stimulation, J Neurol Neurosurg Psychiatry 69 (2000), 278-279.

[15] W.R.S. Gowers, Epilepsy, and other chronic convulsive diseases: their causes, symptoms and treatment, pp. xiv. 309. J. and A. Churchill: London, $1881,8^{\circ}$.

[16] A. Guimond, C.M. Braun, E. Belanger and I. Rouleau, Ictal fear depends on the cerebral laterality of the epileptic activity, Epileptic Disord 10 (2008), 101-112.

[17] J.M.D. Jackson, On Epilepsy; in answer to the question-What is the nature of the internal commotion which takes place during an epileptic paroxysm?, London, $1850,8^{\circ}$.

[18] K. Kanemoto, J. Kawasaki and I. Kawai, Postictal psychosis: a comparison with acute interictal and chronic psychoses, Epilepsia 37 (1996), 551-556.

[19] K. Kanemoto, J. Kawasaki and E. Mori, Postictal psychosis as a risk factor for mood disorders after temporal lobe surgery, $J$ Neurol Neurosurg Psychiatry 65 (1998), 587-589.

[20] K. Kanemoto, J. Kawasaki and E. Mori, Violence and epilepsy: a close relation between violence and postictal psychosis, Epilepsia 40 (1999), 107-109.

[21] A.M. Kanner and J.J. Barry, Is the Psychopathology of Epilepsy Different from That of Nonepileptic Patients? Epilepsy Behav 2 (2001), 170-186.

[22] A.M. Kanner and A. Ostrovskaya, Long-term significance of postictal psychotic episodes I. Are they predictive of bilateral ictal foci? Epilepsy Behav 12 (2008), 150-153.

[23] A.M. Kanner and A. Ostrovskaya, Long-term significance of postictal psychotic episodes II. Are they predictive of interictal psychotic episodes? Epilepsy Behav 12 (2008), 154-156.

[24] A.M. Kanner and S.C. Schachter, Psychiatric controversies in epilepsy, Elsevier/Academic Press, San Diego, Calif.; London, 2008, xiv, 327.

[25] A.M. Kanner, A. Soto and H. Gross-Kanner, Prevalence and clinical characteristics of postictal psychiatric symptoms in partial epilepsy, Neurology 62 (2004), 708-713.

[26] E. Kraepelin and A.R. Diefendorf, Clinical psychiatry (1907), Scholars' Facsimiles and Reprints, Delmar, N.Y., 1981, xvii, $562 \mathrm{p}$.
[27] H. Landolt, [Psychic disorders in epilepsy. Clinical and electroencephalographic research.], Dtsch Med Wochenschr 87 (1962), 446-452.

[28] S.J. Logsdail and B.K. Toone, Post-ictal psychoses. A clinical and phenomenological description, Br J Psychiatry 152 (1988), 246-252.

[29] M. Mula, R. Jauch, A. Cavanna, V. Gaus, R. Kretz, L. Collimedaglia, D. Barbagli, R. Cantello, F. Monaco and B. Schmitz, Interictal dysphoric disorder and periictal dysphoric symptoms in patients with epilepsy, Epilepsia 51 (2010), 1139-1145.

[30] M. Mula, B. Schmitz, R. Jauch, A. Cavanna, R. Cantello, F. Monaco and M.R. Trimble, On the prevalence of bipolar disorder in epilepsy, Epilepsy Behav 13 (2008), 658-661.

[31] S. Nadkarni, V. Arnedo and O. Devinsky, Psychosis in epilepsy patients, Epilepsia 48(Suppl 9) (2007), 17-19.

[32] T. Nishida, T. Kudo, Y. Inoue, F. Nakamura, M. Yoshimura, K. Matsuda, K. Yagi and T. Fujiwara, Postictal mania versus postictal psychosis: differences in clinical features, epileptogenic zone, and brain functional changes during postictal period, Epilepsia 47 (2006), 2104-2114.

[33] T. Oshima, Y. Tadokoro and K. Kanemoto, A prospective study of postictal psychoses with emphasis on the periictal type, Epilepsia 47 (2006), 2131-2134.

[34] G. Savard, F. Andermann, A. Olivier and G.M. Remillard, Postictal psychosis after partial complex seizures: a multiple case study, Epilepsia 32 (1991), 225-231.

[35] A. Scaramelli, P. Braga, A. Avellanal, A. Bogacz, C. Camejo, I. Rega, T. Messano and B. Arciere, Prodromal symptoms in epileptic patients: clinical characterization of the pre-ictal phase, Seizure 18 (2009), 246-250.

[36] D.B. Smith, D.M. Treiman and M.R. Trimble, Neurobehavioral problems in epilepsy, Raven, New York, 1991, 485.

[37] H. Tellenbach, [Epilepsy as a Convulsive Disorder and as a Psychosis. On Alternative Psychoses of Paranoid Nature in "Forced Normalization" (Landolt) of the Electroencephalogram of Epileptics.], Nervenarzt 36 (1965), 190-202.

[38] V. Toth, A. Fogarasi, K. Karadi, N. Kovacs, A. Ebner and J. Janszky, Ictal affective symptoms in temporal lobe epilepsy are related to gender and age, Epilepsia 51 (2010), 1126-1132.

[39] M.R. Trimble, The psychoses of epilepsy, Raven Press, New York, 1991.

[40] M.R. Trimble and B. Schmitz, Forced normalization and alternative psychoses of epilepsy, Wrightson Biomedical Pub. Ltd., Petersfield, 1998, xi,235p. 


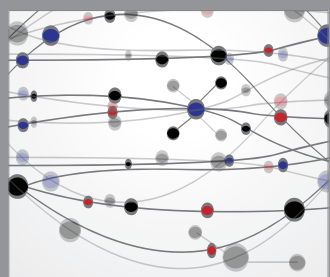

The Scientific World Journal
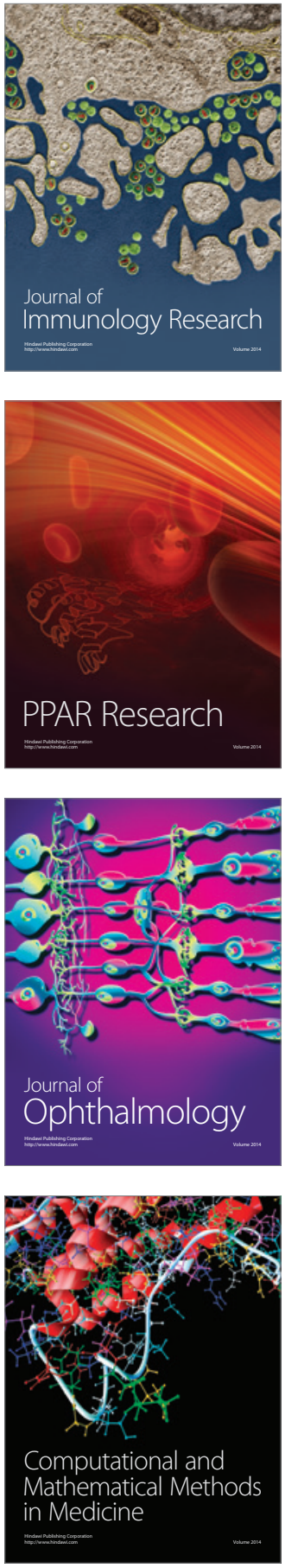

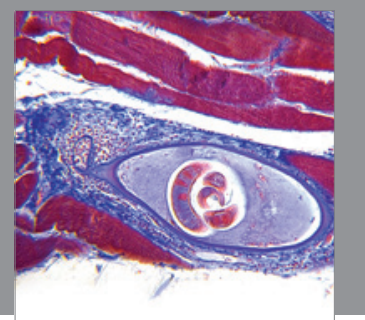

Gastroenterology

Research and Practice
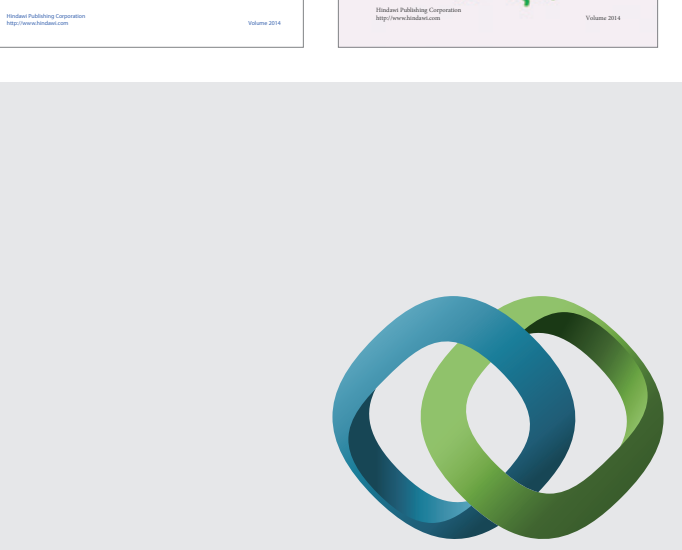

\section{Hindawi}

Submit your manuscripts at

http://www.hindawi.com
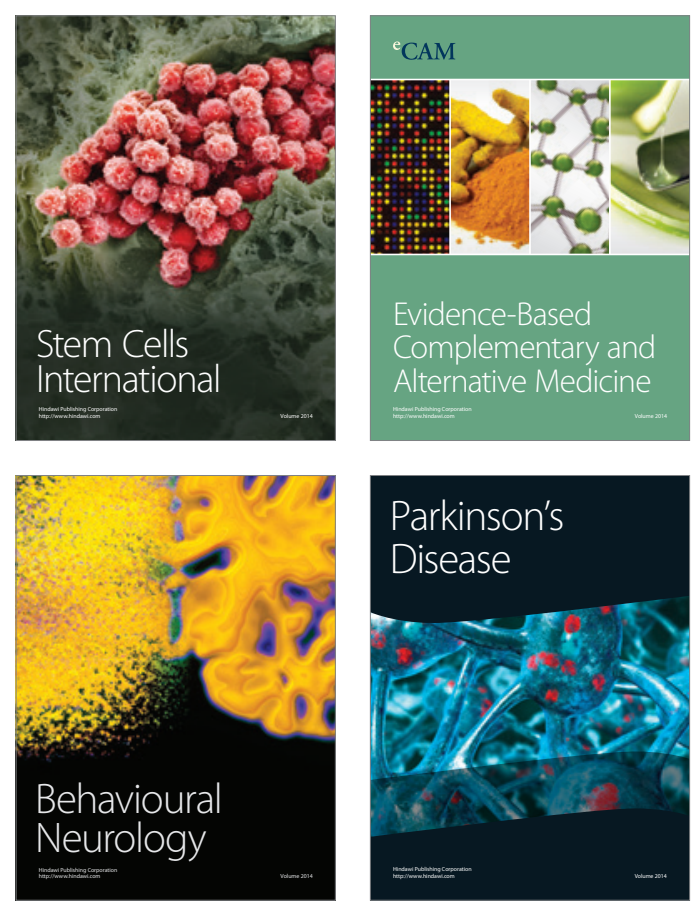

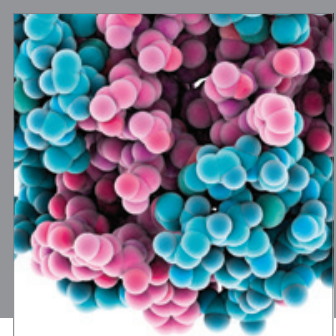

Journal of
Diabetes Research

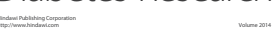

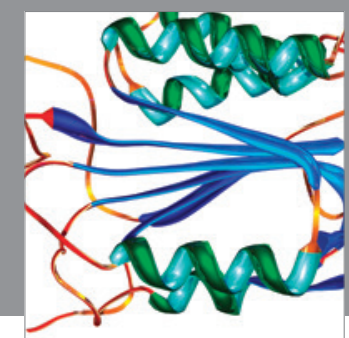

Disease Markers
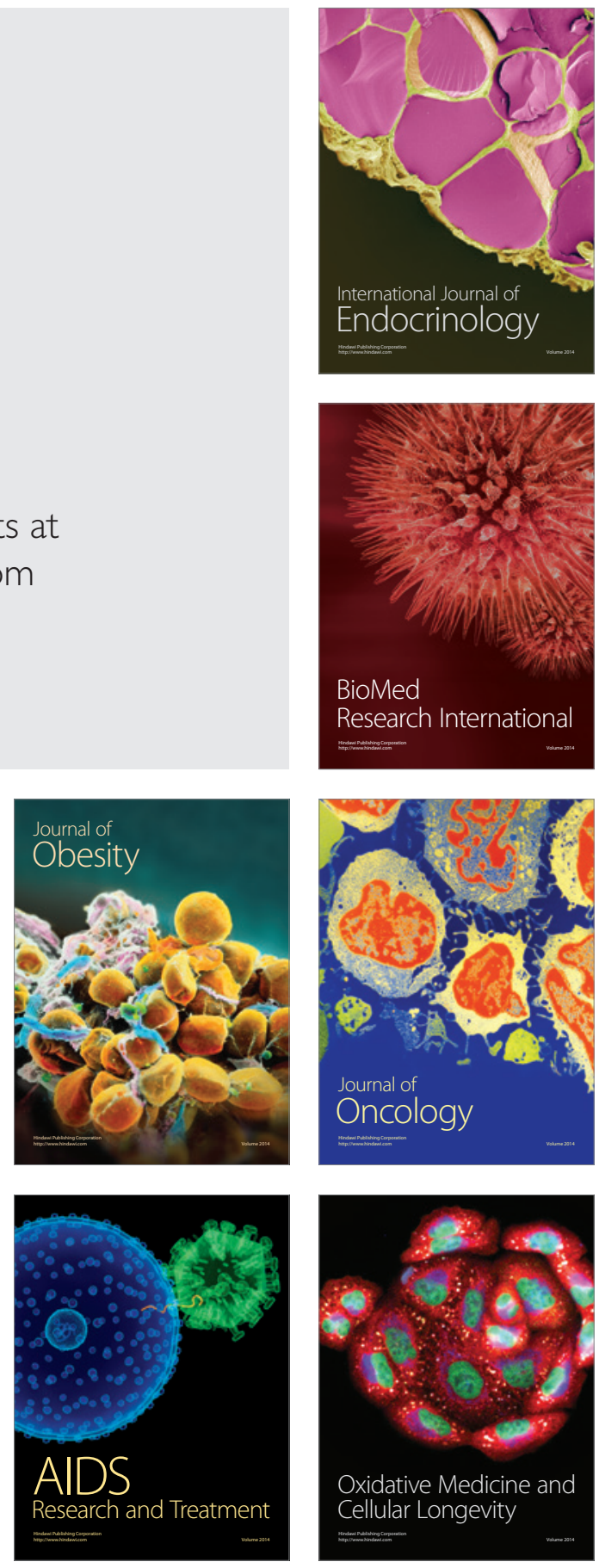